\title{
Secondary Cerebrovascular Prevention in Light of the COVID-19 Pandemic
}

\section{$G$ Padlina, MD}

$S$ Bellwald, MD

\section{A Maurer, MD}

MR Heldner, MD, MSC ${ }^{*} \odot$

\author{
Address \\ "Department of Neurology, Inselspital, University Hospital and University of Bern, \\ Bern, Switzerland \\ Email: mirjam.heldner@insel.ch
}

Published online: 5 August 2020

(C) Springer Science+Business Media, LLC, part of Springer Nature 2020

This article is part of the Topical Collection on Reflections from the COVID Pandemic

Keywords Secondary cerebrovascular prevention - COVID-19

\section{Abstract}

Background: The COVID-19 pandemic has affected healthcare systems, professionals and patients around the world. At the same time, the burden of cerebrovascular events is considerable. Worldwide, more than one million deaths per year are due to cerebrovascular events, which are the second most frequent cause of death and the main cause of long-term disability in Europe. To approach the challenges of the COVID-19 pandemic and secondary cerebrovascular prevention:

Conclusion: We recommend:

(1) As in normal times, during the COVID-19 pandemic wave, patients need to seek urgent medical attention in case of any acute cerebrovascular event. This will assure they receive needed rapid cerebrovascular secondary prevention in addition to acute intravenous and endovascular reperfusion strategies.

(2) As in normal times, during the COVID-19 pandemic wave, it is of utmost importance that patients adhere to their individual recommendations for secondary prevention.

(3) Optimal secondary but also primary cerebrovascular prevention might reduce the burden of COVID-19 now and during potential subsequent waves.

(4) Patients with cerebrovascular disease should strictly adhere to advice concerning the pandemic provided by their governments and to pandemicrelated recommendations for them as individuals expressed by their treating physicians. 
"Secondary cerebrovascular prevention patients are highly impacted by the COVID-19 pandemic and at the same time are impacting hospital capacities due to their own disability, morbidity and mortality."

\section{Introduction}

The burden of cerebrovascular events is considerable. Worldwide, more than one million deaths per year are due to cerebrovascular events. These events are the second most frequent cause of death and the main cause of long-term disability in Europe [1, 2].

After a cerebrovascular event, besides acute intravenous and/or endovascular reperfusion strategies, rapid secondary prevention at a stroke unit or centre is crucial to improve outcome and to lower the recurrence risk of any vascular event in the short term and also in the long term. A recurrent vascular event likely increases disability and mortality and could be prevented in around $80 \%$ of patients. Rapid secondary prevention includes investigation and therapy of cerebrovascular event aetiology, prescription of medications, treatment of vascular risk factors, and adaptations of lifestyle. After discharge from hospital, ongoing regular doctor patient contacts ensure adequacy and efficacy of treatment strategies and facilitate persisting adherence [3].

Our way of providing cerebrovascular secondary prevention at the neurovascular laboratory at the
Inselspital, University Hospital of Bern/Switzerland, has been established and adapted over years. However, this has been disrupted by the COVID-19 pandemic. After a shift of the epicentre from China to Europe, Switzerland soon showed and still shows one of the highest numbers of total patients testing positive per 1 million population. From mid-march 2020, the Swiss government declared a soft lockdown which finally lasted for 8 weeks at its full extent (https://www. swissinfo.ch/eng/covid-19_coronavirus\%2D\%2Dthesituation-in-switzerland/45592192). Within a few days, our service at the neurovascular laboratory had to adjust to the new circumstances in which healthcare systems and professionals started to prioritize care to COVID-19 and intermediate/intensive/emergency care.

Normally, we offer cerebrovascular secondary prevention for patients in both in- and outpatient settings. The service provides consultations and comprehensive diagnostics. Neurosonography of brainsupplying arteries reveals pathologies such as vessel

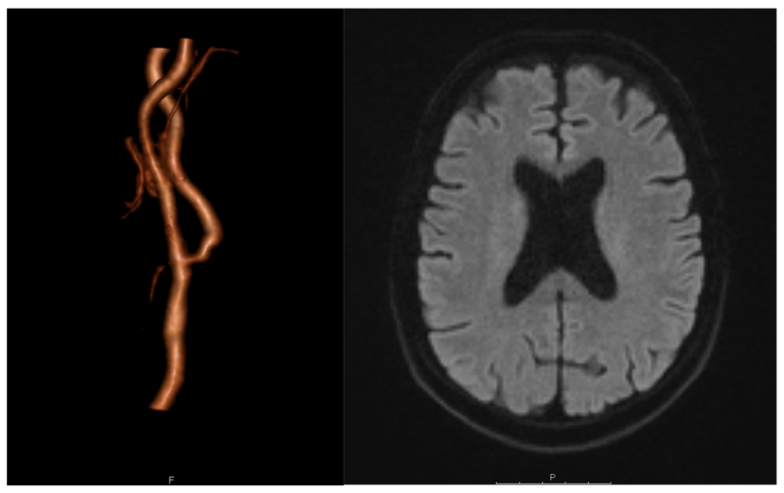

a

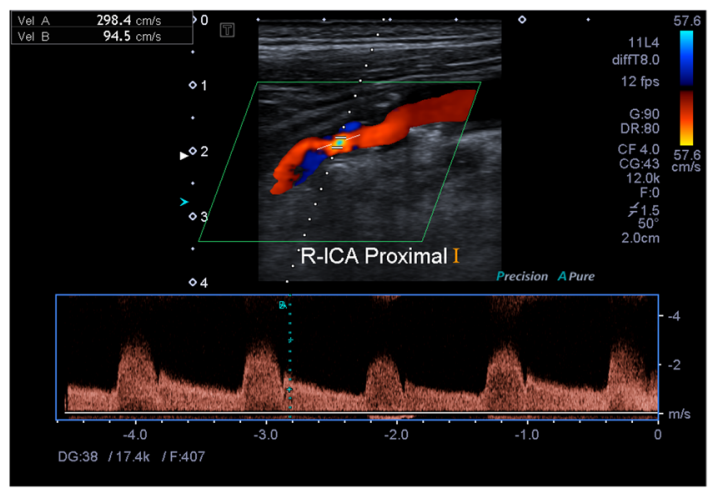

b

Fig. 1. A 70-year old patient suffered an amaurosis fugax on the right side lasting for some minutes. On admission, his NIHSS score was 0. MR imaging (a) and neurosonography (b) revealed a moderate stenosis of the extracranial internal carotid artery on the right side due to atherosclerosis without any DWI lesion. He was obese, smoking and had arterial hypertension with hypertensive cardiomyopathy and coronary heart disease. Cardiac rhythm monitoring on the stroke unit did not reveal any relevant arrhythmia. Daily aspirin $100 \mathrm{mg}$ and atorvastatin $80 \mathrm{mg}$ were initiated and a carotid endarterectomy of the extracranial internal carotid artery on the right side performed on day 4. There was no complication. He was discharged home without any focal neurological deficits on day 6 . He has been adhering to medications and has adapted his lifestyle since. No recurrent event has occurred for 1 year by now. 
occlusions, stenoses (Fig. 1) or dissections that are correlated with other neuroimaging findings (multimodal magnetic resonance and/or computer tomography and/or digital subtraction angiography) performed at our tertiary stroke centre. Potential cardioembolism is investigated by bubble test and transcranial Doppler monitoring for detection of microemboli.

\section{Points to Consider in Secondary Cerebrovascular Prevention in Light of the COVID-19 Pandemic}

(1) Over the past weeks, many colleagues around the world have noted fewer patients with cerebrovascular events presenting at hospitals, especially fewer with mild to moderately disabling disease. There is no rationale to suggest that this is due to a decrease of incidence of cerebrovascular events during the COVID-19 pandemic (https://esostroke.org/likely-increase-in-the-risk-of-death-ordisability-from-stroke-during-the-covid-19pandemic/). Likely explanations are that patients are not seeking medical attention because of fear of being infected with COVID-19 in the hospital, they are assuming that healthcare professionals are too busy during the pandemic to treat them, they are strictly adhering to lockdown policies, and/or because their symptoms are not self-recognized and family members are not on site to notice.

(2) In a survey by the European Stroke Organisation (ESO) among 426 stroke care providers from 55 countries, only around $20 \%$ reported that patients were continuing to receive usual secondary cerebrovascular prevention. In several countries, stroke care pathways were modified, and staff was redeployed to COVID-19 wards, intermediate/intensive care, internal medicine, pneumology and/or to emergency units. Outpatient clinics and elective procedures were suspended, creating shortages for dedicated secondary prevention services (https:// eso-stroke.org/likely-increase-in-the-risk-of-deathor-disability-from-stroke-during-the-covid-19pandemic/) [4].

(3) The majority of patients with cerebrovascular disease are older than 60 years of age, some considerably so. Many patients with cerebrovascular disease suffer from one or several vascular risk factors. Older patients and/or those with a vascular risk factor profile-especially those with previous vascular events, arterial hypertension, diabetes mellitus and/or obesity-have been demonstrated to have a considerable risk to be infected with COVID-19 and to present with severe disease, needing intensive care admission and showing poor outcome [5-12].

(4) According to the INTERSTROKE study, arterial hypertension, physical inactivity, being overweight, dyslipidaemia, smoking, unhealthy diet, cardiac pathologies, diabetes mellitus, stress/depression and overconsumption of alcohol are the most important modifiable vascular risk factors [13]. How these vascular risk factors and their treatment impact COVID-19 is a matter of investigation.

There is controversy about antihypertensive medications having a possible adverse, protective or biphasic effect in patients with COVID-19. Angiotensin-converting enzyme (ACE) inhibitors and angiotensin receptor blockers increase levels of ACE 2. Physiologically, ACE 2 contributes to protect from lung injury and heart failure. But SARS-CoV-2, which causes COVID-19, uses docking to ACE 2 to enter human cells. This leads to a partial decrease in ACE 2 function and thereby to dominant angiotensin II effects. Angiotensin II triggers inflammation, cell proliferation, hypertrophy, fibrosis and tissue remodeling. Therefore, ACE inhibitors and angiotensin receptor blockers could hypothetically be beneficial by antagonizing some detrimental effects of SARS-CoV-2 but might also increase the risk of infection as well as the severity of COVID-19 by increasing ACE 2 binding sites. At present, guidelines recommend to continue these antihypertensive medications in non-infected patients and patients at risk, not to switch patients from an established to a new regime, and not to initiate their prophylactic use in healthy people at risk [14].

Lockdown, social distancing and economic downturn are changing lifestyle, dietary and smoking habits, are reducing physical activity and are increasing obesity and vascular risk factor profile in some people $[9,12]$. Also, lockdown, rapid transmission of COVID-19, high number of deaths, lack of effective treatment strategies and vaccines have led to mental health problems such as fear, anxiety, depression, stress reactions, posttraumatic stress disorder and sleep problems [15]. 
(5) Infections have previously been identified to increase risk of cerebrovascular events. This appears to hold true for COVID-19 as well. Mechanisms of increasing the risk of cerebrovascular events may be endothelial dysfunction, hypercoagulability, hypoxia, myocardial infarction, heart failure, cardiac arrhythmia, vasculitis and/or plaque instability and rupture [5-8, 16-21].

(6) In recent years, governments have often invested far more in acute care than in secondary and primary cerebrovascular prevention [3]. This has contributed to higher risk factor profiles and higher morbidity in ageing populations.

(7) Several countries have reported high rates of healthcare professionals contracting COVID-19 [22].

To summarize, patients cared for in neurovascular programmes are a special high-risk group of patients in light of COVID-19, and furthermore, COVID-19 is affecting staff as well. To address this challenge, the neurovascular laboratory at the Inselspital, University Hospital of Bern/Switzerland, took the following steps:

\section{Reorganization of the Neurovascular Laboratory During the Peak of the Current Wave of the COVID-19 Pandemic}

The following steps were taken:

(1) The staff was split into teams A and B. Team A was working in the hospital for 1 week, while team B was working from home as a back-up team. The week thereafter, the teams switched. Our hospital management implemented this strategy in all departments in order to deal with potential illness/quarantine measures and to continue providing specialist services to some extent.

(2) Normally, our neurovascular laboratory is staffed by three stroke consultants, one stroke fellow, three junior doctors and one visiting doctor, two technicians and two secretaries. Workforce optimization reduced the staff to two stroke consultants, two junior doctors, two technicians and two secretaries, half of which were allocated to team A and B respectively. The other team members were redeployed within our hospital.

(3) Neurosonography examinations were limited to a few essential/emergency indications such as symptomatic carotid stenosis, vasculitis or floating thrombi/plaques.

(4) Only essential/emergency consultations were done face to face with the patient.

(5) The majority of scheduled, in person visits were cancelled and carried out by phone or telemedicine.

(6) Phone consultations were carried out in additional selected patients with a high-risk of morbidity and mortality from COVID-19. These were patients who were already being followed by the neurovascular laboratory (for several months or years) at regular intervals (e.g., patients with chronic stenosis or occlusions of the brainsupplying arteries).

(7) Reports of these consultations were sent to referring physicians, to the patients themselves and/or to the people supporting them.

(8) We adjusted and guaranteed ongoing prescriptions and deliveries of medications.

(9) For all contacts with the patients and/or people supporting them, we did our best to provide thoughtful problem solving. We provided education on the importance of secondary cerebrovascular prevention and of calling for medical help in case of any acute cerebrovascular event and/or any other emergency. We also discussed the COVID-19 pandemic and pandemic-related recommendations for them as individuals. Patients and caregivers were referred to web-based information and information leaflets. Self-home monitoring of vascular risk factors and a healthy lifestyle were promoted and activity-based training instructions were provided.

(10) The weekly neurovascular board meeting was replaced by regular phone calls among the specialists.

(11) A new digital work sheet was established in which responsibilities and workload was divided among the staff.

(12) Regular web-based meetings were performed in which information was exchanged about latest developments of the COVID-19 pandemic, current steps hospital management was taking and about suggested organization of hospital services. 
(13) Phone calls to the neurovascular laboratory staff were routed to the staff members' personal phones by an app that maintained the privacy of the personal phone number.

(14) Letters and correspondence were not signed by hand anymore, but by ticking boxes digitally.

(15) We were digitalizing all our neurosonography images, flow velocities and plaque characteristics on a regular basis allowing for remote access, which was needed more than in normal times by the staff.

(16) In-hospital, staff as well as patients strictly adhered to rules of conduct (e.g., wearing protective equipment, paying attention to hygiene and disinfection of equipment, social distancing, limited point of contact) as advised and continuously adapted by the hospital management.

\section{Conclusion}

By implementing new protocols and procedures, and using innovative technological adaptations, we have been able to continue providing specialist service in our neurovascular laboratory during the peak of the current COVID-19 pandemic wave. We have experienced gratitude by patients and people caring for them. Our individual and collective performance at work saw a boost of empathy, efficiency, focus, prioritization, calmness, resilience, team spirit, creativeness, motivation and openness to flexibility and unpredictability.

(1) As in normal times, during the COVID-19 pandemic wave, patients need to seek urgent medical attention in case of any acute cerebrovascular event. This will assure they receive needed rapid cerebrovascular secondary prevention in addition to acute intravenous and endovascular reperfusion strategies.

(2) As in normal times, during the COVID-19 pandemic wave, it is of utmost importance that patients adhere to their individual recommendations for secondary prevention.

(3) Optimal secondary but also primary cerebrovascular prevention might reduce the burden of COVID-19 now and during potential subsequent waves.

(4) Patients with cerebrovascular disease should strictly adhere to advice concerning the pandemic provided by their governments and to pandemic-related recommendations for them as individuals expressed by their treating physicians.

\section{Conflict of interest}

Giovanna Padlina has nothing to disclose.

Sebastian Bellwald has nothing to disclose.

Alexander Maurer has nothing to disclose. 
Mirjam R Heldner has served on the Advisory Board of Amgen in 2019 and in 2020, outside the submitted work.

\section{References and Recommended Reading}

1. Lozano R, Naghavi M, Foreman K, Lim S, Shibuya $\mathrm{K}$, Aboyans $\mathrm{V}$, et al. Global and regional mortality from 235 causes of death for 20 age groups in 1990 and 2010: a systematic analysis for the global burden of disease study 2010. Lancet. 2012;380:2095128 .

2. GBD 2016 Stroke Collaborators. Global, regional, and national burden of stroke, 1990-2016: a systematic analysis for the Global Burden of Disease Study 2016. Lancet Neurol. 2019;18:439-58.

3. Webb A, Heldner MR, Aguiar de Sousa D, et al. Availability of secondary prevention services after stroke in Europe: an ESO/SAFE survey of national scientific societies and stroke experts. Eur Stroke J. 2019;4:110-8.

4. Zhao J, Rudd A, Liu R. Challenges and potential solutions of stroke care during the coronavirus disease 2019 (COVID-19) outbreak. Stroke. 2020;51:1356-7.

5. Wu Z, McGoogan JM. Characteristics of and important lessons from the coronavirus disease 2019 (COVID19) outbreak in China: summary of a report of 72314 cases from the Chinese Center for Disease Control and Prevention. JAMA. 2020 Feb 24. Online ahead of print.

6. Yang J, Zheng Y, Gou X, et al. Prevalence of comorbidities in the novel Wuhan coronavirus (COVID-19) infection: a systematic review and meta-analysis. Int J Infect Dis. 2020;94:91-95.

7. Huang C, Wang Y, Li X, et al. Clinical features of patients infected with 2019 novel coronavirus in Wuhan, China. Lancet. 2020;395:497-506.

8. Clerkin KJ, Fried JA, Raikhelkar J, et al. Coronavirus disease 2019 (COVID-19) and cardiovascular disease. Circulation. 2020;141:1648-1655.

9. Sattar N, McInnes IB, McMurray JJV. Obesity a risk factor for severe COVID-19 infection: multiple potential mechanisms. Circulation. 2020;142:4-6.

10. Huang I, Lim MA, Pranata R. Diabetes mellitus is associated with increased mortality and severity of disease in COVID-19 pneumonia - a systematic review, meta-analysis, and meta-regression. Diabetes Metab Syndr. 2020;14:395-403.

11. Richardson S, Hirsch JS, Narasimhan M, the Northwell COVID-19 Research Consortium, et al. Presenting characteristics, comorbidities and outcomes among 5700 patients hospitalized with COVID-19 in the New York city area. JAMA. 2020;323:2052-2059.
12. Duffy EY, Cainzos-Achirica M. Michos ED. Circulation: Primary and secondary prevention of cardiovascular disease in the era of the coronavirus pandemic; 2020.

13. O'Donnell MJ, Xavier D, Lisheng L, et al. Risk factors for ischaemic and intracerebral haemorrhagic stroke in 22 countries (the INTERSTROKE study): a case control study. Lancet. 2010;376:112-23.

14. Messerli FH, Siontis GCM, Rexhaj E. Covid-19 and renin angiotensin blockers: current evidence and recommendations. Circulation. 2020;141:2042-2044.

15. Xiang YT, Jin Y, Cheung T. Joint international collaboration to combat mental health challenges during the coronavirus disease 2019 pandemic. JAMA Psychiatry. 2020 Apr 10. Online ahead of print.

16. Smeeth L, Thomas SL, Hall AJ, Hubbard R, Farrington $\mathrm{P}$, Vallance P. Risk of myocardial infarction and stroke after acute infection or vaccination. NEJM.

2004;351:2611-8.

17. Elkind MSV. Why now? Moving from stroke risk factors to stroke triggers. Curr Opin Neurol. 2007;20:51-7.

18. Oxley TJ, Mocco J, Majidi S, et al. Large-vessel stroke as a presenting feature of COVID-19 in the young. NEJM. 2020;382:e60.

19. Li Y, Wang M, Zhou Y, et al. Acute cerebrovascular disease following COVID-19: a single center, retrospective observational study. Lancet. 2020Stroke and Vascular Neurology. 2020;svn-2020-000431.

20. Lazzerini PE, Boutjdir M, Capecchi PL. COVID-19, arrhythmic risk and inflammation: mind the gap! Circulation. 2020

21. Mao L, Wang M, Chen S, et al. Neurological manifestations of hospitalized patients with COVID-19 in Wuhan, China: a retrospective case series study. JAMA Neurol. 2020;77:1-9.

22. Harkin DW. Covid-19: balancing personal risk and professional duty. BMJ. 2020;369:m1606.

\section{Publisher's Note}

Springer Nature remains neutral with regard to jurisdictional claims in published maps and institutional affiliations. 